\title{
Wnt/ß-catenin signalling plays diverse functions during the process of fibrotic remodelling in the exocrine pancreas
}

Merja Bläuer, Matias Laaninen, Juhani Sand, Johanna Laukkarinen ${ }^{1}$

Tampere Pancreas Laboratory and Department of Gastroenterology and Alimentary Tract Surgery, Tampere University Hospital and Faculty of Medicine and Life Sciences, Tampere University, Tampere, Finland

SHORT TITLE: Wnt/ $\beta$-catenin expression in pancreatic fibrosis

${ }^{1)}$ Corresponding author: Department of Gastroenterology and Alimentary Tract Surgery, Tampere University Hospital, Teiskontie 35, FIN-33521 Tampere, Finland; Tel.: +358 3 311611; fax: +358 33116 4358; E-mail address: johanna.laukkarinen@fimnet.fi (J.Laukkarinen) 
Abstract

Background/Objectives: Wnt/ $\beta$-catenin signalling plays vital roles in tissue homeostasis. Dysregulation of the pathway has been implicated in the pathogenesis of cancer and fibroses in numerous tissues, including the pancreas. We studied the effect of microenvironmental changes pertaining to fibrotic tissue remodelling on the expression of selected Wnt/ $\beta$ catenin pathway proteins in the human exocrine pancreas. The role of acinar/stellate cross-talk on the expression of the proteins was elucidated in a long-term mouse co-culture system.

Methods: Expression of $\beta$-catenin, Wnt2, Wnt5a and SFRP4 was analysed immunohistochemically in normal and moderately or highly fibrotic human pancreata $(n=8)$. The effect of humoral interactions on the expression of the proteins was studied by immunocytochemical means in parallel mono- and co-cultures of mouse acinar and stellate cells (PSCs).

Results: In human pancreatic tissue, fibrotic microenvironment was associated with redistribution of the proteins in and between epithelial and stromal compartments, compared to acinar-rich tissue. In non-fibrotic and moderately fibrotic tissue the proteins appeared only in acinar cells whereas in highly fibrotic tissue stromal fibroblastoid/stellate cells and macrophages were their predominant locations. Subcellular changes in the expression of $\beta$-catenin and Wnt5a were detected. Our in vitro data suggest potential involvement of acinar cell/PSC cross-talk in mediating the changes observed in tissue specimens.

Conclusions: Wnt/ $\beta$-catenin pathway-associated proteins are abundantly expressed in the exocrine pancreas with prominent changes in their cellular and subcellular expression patterns along with increasing levels of fibrosis. Diverse functions for $\mathrm{Wnt} / \beta$-catenin signalling during the course of fibrotic remodelling in the exocrine pancreas are suggested.

Keywords: acinar cell, co-culture, fibrosis, in vitro, stellate cell 


\section{Introduction}

The Wnt/ $\beta$-catenin signalling cascade is an evolutionary conserved pathway that plays a fundamental role in directing morphogenesis during embryonic development and maintaining tissue homeostasis in adult organisms [1,2].

Dysregulation of the pathway has been implicated in developmental abnormalities as well as the pathogenesis of various proliferative diseases, such as cancer and fibroses [1-4].

Central components of the Wnt/ $\beta$-catenin pathway include Wnt ligands, the Frizzled (Fzd) family of membrane receptors and $\beta$-catenin $[1,2]$. In canonical Wnt/ $\beta$-catenin signalling, binding of a Wnt ligand to Fzd assigns $\beta$-catenin to translocate from the cytoplasm to the nucleus to modulate transcription. Besides gene regulation, $\beta$-catenin plays a central role as a structural component of adherens junctions. The coordination and interchangeability of the two roles are, as yet, not completely understood $[1,2,5]$. Some of the 19 Wnts identified in vertebrates also operate in noncanonical pathways independently of $\beta$-catenin. While mainly activating the non-canonical signalling cascades, such Wnts can also either inhibit or activate the canonical Wnt/ $\beta$-catenin pathway, depending on the cellular context [6-8]. Further regulation to Wnt/ $\beta$-catenin signalling is afforded by a wide range of intra- and extracellular agonists and antagonists [9], including the secreted frizzled-related protein (SFRP) family of Wnt inhibitors [10].

Transcripts of a vast array of Wnt $/ \beta$-catenin pathway-associated factors have been detected in the endocrine and exocrine compartments of the adult human pancreas [11]. They have been shown to take part in normal pancreas development, growth and regeneration [12], and their involvement in the pathogenesis of chronic pancreatitis and pancreatic adenocarcinoma has been suggested [13]. Both autocrine [14,15] and paracrine [16-18] signalling mechanisms for $\mathrm{Wnt} / \beta$-catenin have been described in pancreatic cancer, the latter being mediated by stromal cells. Moreover, SFRP4 produced by PSCs has been shown to modulate the cancer microenvironment $[16,19]$.

Pancreatic stellate cells (PSCs) are central contributors to the fibrotic remodelling of the stromal compartment in pancreatitis and cancer [20]. Their persistent activation in chronic pancreatitis has been associated with aberrant regulation of Wnt/ $\beta$-catenin signalling [21,22]. Suppression of Wnt/ $\beta$-catenin signalling in PSCs has been shown to inhibit their activation and to ameliorate fibrosis in pancreatic diseases [16,22].

In addition to displaying specific individual functions, the $\mathrm{Wnt} / \beta$-catenin pathway is known to act cooperatively with other regulatory pathways such as those of TGF- $\beta$ and $N F \kappa \beta[23,24]$. Both these signalling systems have previously been implicated in pancreatic inflammation, fibrosis and cancer [25-27]. 
The aim of the present study was to provide further insight into $\mathrm{Wnt} / \beta$-catenin signalling in the exocrine pancreas in relation to fibrotic tissue remodelling. Antibodies against a set of proteins associated with the Wnt/ $\beta$-catenin pathway were used to compare their cellular and subcellular expression in non-fibrotic and fibrotic human tissue samples. The targets of interest included $\beta$-catenin, Wnt2 and Wnt5a representing canonical and non-canonical ligands, respectively, and SFRP4. The effect of cross-talk between acinar cells and PSCs on the expression of the antigens was examined using the long-term mouse co-culture technique developed in our laboratory [28].

\section{Materials and methods}

\section{Human pancreatic tissue}

Tissue specimens were taken from the tumour-free resection line of pancreata from subjects undergoing pancreatic surgery at the Tampere University Hospital, fixed in 4\% formaldehyde and embedded in paraffin. The indications for pancreatic resection were adenocarcinoma $(n=7)$ and serous cystic neoplasm $(n=1)$. According to histological structure, three non-fibrotic tissues and five samples with varying degrees of fibrosis were proceeded for immunohistochemical analyses. One of the latter represented moderate and four highly fibrotic histology. The use of human pancreatic tissue for research purposes was approved by the Ethics Committee of Tampere University Hospital (decision number R07209).

Mono- and co-culture of mouse acinar cells and PSCS

Pancreatic acinar cells and PSCs were obtained from healthy mice of the strain c57BL/6JOlaHsd (Harlan, the Netherlands). Approval for the use of mice as donors of pancreatic tissue for in vitro experiments was obtained from the Institutional Animal Welfare Committee. Acinar cells and PSCs were prepared and cryopreserved for on demand use as previously described [29,30]. After thawing, monocultures of each cell type and parallel co-cultures were established using the experimental protocol introduced by Bläuer et al. [28]. Acinar-to-PSC ratio at the onset of coculture was approximately $1: 1$. The cultures were maintained for 4 days at $37^{\circ} \mathrm{C}$ in a humidified atmosphere of $5 \% \mathrm{CO}_{2}$ in air and half the volume of the culture medium was renewed every day. The cultures were terminated by fixation in $2 \%$ formaldehyde.

\section{Immunohisto- and cytochemistry}

After deparaffination and antigen unmasking in citrate buffer (pH 6.0) $5 \mu \mathrm{m}$-thick tissue slices of the human pancreatic samples were probed with rabbit primary antibodies against selected $\mathrm{Wnt} / \beta$-catenin pathway proteins. The selection 
was made based on previous studies on their involvement in pancreatic physiology and/or pathophysiology [11,13,16,21,22]. Anti- $\beta$-catenin (ab16051), anti-Wnt2 (ab109222) and anti-SFRP4 (ab154167) antibodies were purchased from Abcam, Cambridge, UK and the one against Wnt5a (PA5-72454) was from Thermo Fisher Scientific, Rockford, IL. Antibody dilutions were 1:100 for anti- $\beta$-catenin and anti Wnt2 and 1:500 and 1:50 for the antibodies against Wnt5a and SFRP4, respectively. The staining was performed with the Histostain Plus Broad Spectrum kit from Life Technologies, Frederick, MD, followed by chromogenic detection using diaminobenzidine (DAB) as the substrate (DAB Substrate Kit, Thermo Fisher Scientific).

The above primary antibodies with the same dilutions (except for SFRP4 1:100) were also used for staining the monoand co-cultures of mouse pancreatic acinar and PSCs. Before commencing the immunocytochemical procedure as previously described [28], the cells were permeabilized with $94 \%$ ethanol.

Negative controls for immunohisto- and cytochemical stainings were performed by substituting plain antibody dilution medium for the primary antibodies (Supplement 1). Four human carcinoma cell lines including MCF-7 (mammary), PANC-1 (pancreas) and VCaP and PC-3 (prostate) together with our in-house primary epithelial cell line P100E originating from human prostatic hyperplasia were used to validate the antibodies in Western blotting and immunocytochemical analyses (Supplement 2). The analyses showed that the antibodies were able to detect their corresponding antigens by both methods.

\section{Quantitation}

Differences in the localization or intensity of immunopositive staining between non-fibrotic and moderately fibrotic tissue areas as well as between mono- and co-cultures were quantitated. Quantitation was performed only on stainings permitting objective differentiation between positivity vs negativity or high vs low intensity. These included nuclear $\beta$ catenin and Wnt5a in human tissues, nuclear Wnt5a and cytoplasmic SFRP4 in mouse acinar cells, and nuclear $\beta$ catenin and cytoplasmic SFRP4 in mouse PSCs. Six randomly picked microscopic areas of tissue sections or of cells in two independent cultures were analysed (200x magnification). In human tissues, cross sections of whole acini were calculated and acini showing at least one immunopositive cell were regarded as positive. The data were presented as mean \pm SEM. 


\section{Results}

Results of the immunohistochemical analyses performed on human pancreatic tissue specimens are presented in Fig 1 and representative immunocytochemical stainings from three independent in vitro experiments are illustrated in Fig 2. A schematic summary of the tissue and in vitro data is depicted in Fig 3 and a graphic presentation of their quantitative analysis is shown in Supplement 3.

\section{Expression Wnt/ $\beta$-catenin pathway-associated proteins in human pancreatic tissue}

All non-fibrotic tissues displaying normal pancreatic tissue architecture were identical in their expression and distribution of the Wnt/ $\beta$-catenin-associated proteins studied (Figs 1a-d). An intense $\beta$-catenin staining was found localized at the acinar cell membrane (Fig la). Acinar cells also showed a strong cytoplasmic expression of SFRP4 (Fig 1d), whereas only a faint positive reaction for both Wnt2 and Wnt5a could be detected (Figs 1b,c). The interacinar stroma of these tissues was negative for all the proteins studied.

Along with the diminishing amount of tissue areas occupied by acinar cells in fibrotic samples, the stroma became the major site of expression of the Wnt/ $\beta$-catenin pathway-associated factors. Moreover, the cellular and subcellular expression of the examined proteins showed greater variability between individual specimens.

Moderate fibrosis in the tissue (Figs $1 e-h)$ resulted in reduced partial or focal membrane staining of $\beta$-catenin in acinar cells (Fig 1e) compared to those in histologically normal tissue (Fig 1a). Positive $\beta$-catenin expression was also observed in scattered nuclei (Fig 1e, arrows) and the cytoplasm of acinar cells together with locally varying intensities of Wnt2 (Fig 1f) and SFRP4 (Fig 1h) immunoreactivities. The percentage of $\beta$-catenin-positive acini (at least one immunopositive nucleus in a cross-section) was $28.2 \pm 2.7 \%$ (mean $\pm \mathrm{SEM}$ ) in moderately fibrotic tissue areas and $3.3 \pm 1.5 \%$ in histologically normal samples $(\mathrm{S} 3)$. In addition to the cytoplasm, Wnt5a was also found localized in numerous (71.7 $\pm 13.4 \%)$ acinar cell nuclei (Fig 1g, inset)

In highly fibrotic tissue areas (Fig 1 i-l) a small number of cells in isolated acinar foci surrounded by dense fibrotic tissue expressed membrane-associated $\beta$-catenin (Fig 1i, open arrow). Strong staining of $\beta$-catenin was present in the fibroblastoid/stellate cells of all non-tumor pancreatic samples from adenocarcinoma patients (Fig $1 i$, arrow) but absent from the tissue obtained from the subject with serous cystic neoplasm. Also the other proteins showed low/nondetectable immunoreactivities in this sample. The intensity of Wnt2 (Fig $1 j$ ) staining in the fibroblastoid/stellate cells of highly fibrotic samples from adenocarcinoma patients was highly variable, while Wnt5a (Fig $1 k)$ remained constantly low. SFRP4 (Fig $1 l$ ) was undetectable in these cells with the exception of one specimen that also showed the 
strongest expression of $\beta$-catenin and Wnts. Wnt2, Wnt5a and SPRP4 expression was undetectable/low in acinar cells surrounded by dense fibrotic tissue areas (Fig lj upper right corner and insets in Figs $1 k$,l).

Macrophages, when present in the fibrotic samples, were shown to intensely express Wnt2, Wnt5a and SFRP4 (Figs 1ik, arrowheads). $\beta$-catenin expression was only detected in few scattered macrophages (Fig 1i, arrowhead).

Expression Wnt/B-catenin pathway-associated proteins in mono-and co-cultures of mouse pancreatic acinar cells and PSCS

The level of expression and the cellular distribution of $\beta$-catenin, Wnt2, Wnt5 and SFRP4 in acinar cells maintained in monoculture (Fig 2a-d) were similar to those observed in non-fibrotic human tissue (Fig 1a-d). Immunoreactive $\beta$ catenin resided predominantly at the cell membrane (Fig 2a) and SFRP4 staining was localized in the cytoplasm (Fig $2 d$ ) with strong expression detected in $14.8 \pm 9.5 \%$ of cells (S3). The expression of both Wnts was at a low level (Figs $2 b, c)$. The number of cells displaying nuclear expression of Wnt5a amounted $3.3 \% \pm 0.0$ of the monocultured acinar cells. In acinar cells co-cultured with PSCs (Figs $2 e-h), \beta$-catenin staining was mainly in the perinuclear cytoplasm (Fig 2e). A faint membrane expression of $\beta$-catenin was seen in areas where cell-cell contacts remained intact (Fig $2 e$ ). No change in Wnt2 was observed in co-cultured acinar cells compared to monoculture (Fig 2f). An approximately 3.6fold increase in the expression level of SFRP4 (Fig 2h) was detected, and the number of cells displaying Wnt5a-positive nuclei increased 6.6-fold compared to monocultured acinar cells (Fig 2g).

In PSC monocultures (Figs 2i-l) $\beta$-catenin was observed in the nuclear and cytoplasmic compartments of the cells (Fig 2i). Nuclear immunopositivity was observed in $29.9 \pm 1.3 \%$ of monocultured PSCs (S3). Wnts (Figs $2 j$, $k$ )were negative or present at a low level while SFRP4 showed strong immunoreactivity in $20.0 \pm 7.1 \%$ of cells. Co-culture with acinar cells (Figs 2m-p, 3e) almost completely abolished nuclear $\beta$-catenin (Fig $2 m$ ). The expression level of both Wnts remained low (Figs 2n,o), while the amount of cells with high expression of SFRP4 increased around 3-fold (Fig 2p).

The summary in Fig 3 schematically demonstrates the observed similarities in the distribution of the Wnt/ $\beta$-catenin pathway associated proteins in acinar cells in non-fibrotic tissues (Fig $3 a$ ) and acinar cell monocultures (Fig 3d). Their redistribution associated with increased fibrosis (Fig 3b) or the presence of PSCs in co-cultures (Fig 3e) is also seen to follow a similar pattern. Reduced expression of Wnt/ $\beta$-catenin pathway-associated proteins is evident in acini and stromal fibroblastoid/stellate cells in highly fibrotic tissue areas (Fig 3c) as well as in monocultured PSCs (Fig 3f). 
Macrophages colonising highly fibrotic tissues, on the other hand, abundantly express the Wnt/ $\beta$-catenin pathwayrelated factors studied (Fig 3c).

\section{Discussion}

Fibrosis in the tissue microenvironment and acinar cell/PSC cross-talk in vitro were shown to evoke prominent changes in the cellular and/or subcellular expression of $\beta$-catenin, Wnt2, Wnt5a and SFRP4 compared to acinar-rich tissud monocultured cells, respectively. The present data suggest diverse functions for the molecules during fibrotic tissue remodelling in the pancreas.

The predominant expression of $\beta$-catenin at the cell membrane of acinar cells in non-fibrotic human pancreatic tissue as well as in mouse acinar cell monocultures agrees with its established role as a structural component of adherens junctions $[1,2]$. That transcriptional $\beta$-catenin signalling may be in a lesser role in normal acinar cell-rich tissue was further supported by the low expression of Wnt2 and the presence of SFRP4 in acinar cells both in vivo and in vitro. It has been shown previously that $\beta$-catenin signalling is not required for the survival and phenotypic maintenance of mature pancreatic acinar cells [13]. Our present data concerning acini in histologically normal human tissue and the dense and functionally adept cell population in acinar cell monocultures $[28,29]$ are in agreement with this observation. $\beta$-catenin seems to be, however, indispensable for the physiological and regenerative proliferation of acinar cells which is known to promote tissue repair e.g. after acute pancreatitis [12,31].

Adjacent fibrosis in the tissue and the presence of PSCs in co-cultures resulted in redistribution of $\beta$-catenin immunoreactivity into the cytoplasm and nuclei of acinar cells, the latter suggesting a switch towards transcription activation. That the ligands stimulating the canonical pathway in moderately fibrotic tissue were most likely provided by the exocrine cells was proposed by the concurrent up-regulation of acinar cell Wnt2. In highly fibrotic tissue, however, these functions appeared to be abolished. In co-cultured acinar cells the observed perinuclear $\beta$-catenin staining was associated with disruption of cell-cell contacts and scattering of the monolayer [28]. Similarly, cell morphology and confluency have previously been observed to control the subcellular localization of $\beta$-catenin in vitro $[32,33]$. The concomitant loss of secretory differentiation of acinar cells in humoral contact with PSCs may also have affected $\beta$-catenin localization in our co-culture environment [28].

Unlike Wnt2, the expression of Wnt5a was strongly increased in co-cultured acinar cells compared to those in monocultures. The detected nuclear accumulation of the molecule suggests a direct non-canonical transcription 
activation in response to PSC-mediated cues. Interestingly, similar subcellular expression pattern for Wnt5a was observed also in acini residing in moderately fibrotic tissue areas. Nuclear Wnt5a has previously been described in the context of various cancers [34-36].

The SFRPs are the largest family of Wnt antagonists [10]. SFRPs resemble the ligand-binding domain of Fzds and are able to extracellularly inhibit ligand-receptor interactions. Ours is the first study to show SFRP4 protein expression in pancreatic acinar cells. In an earlier study using RT-PCR analysis Heller et al. reported SFRP1, 4 and 5 transcripts in the exocrine fraction from an islet isolation procedure [11], their exact cellular source remaining, however, undefined. Besides adding to the tissue pool of pathway inhibitors, the SFRPs produced by acinar cells may be exerting regulatory effects unrelated to Wnt inhibition [10].

Persistent activation of stellate cells is the key event in the development of organ fibrosis in the liver and the pancreas [37]. Wnt/ $\beta$-catenin signalling partakes in this process and its inhibition has been shown to restore stellate cell quiescence $[16,21,22,38]$. Our present data showing up-regulation of Wnt2 and $\beta$-catenin in PSCs in highly fibrotic human tissue samples are in agreement with earlier reports on the roles of Wnt/ $\beta$-catenin signalling in hepatic and pancreatic fibroses $[16,21,22,38]$. Previously imbalanced negative regulation of the pathway in PSCs has been implicated in the etiology of chronic pancreatitis [21] and its suppression has emerged as a potential means to ameliorate pancreatic fibrosis [22]. Our samples displayed individual variation in the expression levels of the molecules, indicating physiological differences in their tissue microenvironment.

We have previously shown that PSCs maintained in co-culture with acinar cells exhibit increased activation compared to monocultures [28]. As activated $\beta$-catenin signalling has been associated with increased activation in PSCs $[16,21,22,38]$, our present observation showing nuclear $\beta$-catenin in PSC monocultures instead of co-cultures was unexpected. PSCs in close contact with each other in the monoculture population were, however, inclined to activate the pathway, most probably by self-produced Wnt ligands. This resembled the expression pattern of the molecules in highly fibrotic tissue areas lacking the paracrine influence of acinar cells. It remains to be examined whether $\beta$-catenin translocation from nuclei to cytoplasm in co-cultures was mediated by the inhibitory action of SFRP4, whose expression in acinar cells as well as PSCs increased in the co-culture setting. As an indication of SFRP-mediated regulation in PSCs, Carapuca et al. [19] showed that in vitro induction of PSC quiescence was associated with enhanced secretion of SFRP4 which, in turn, reduced nuclear $\beta$-catenin in the cells. Hypermethylation or loss of SFRP4 have been associated with the pathogenesis of pancreatic cancer [39,40], further underscoring the importance of the molecule for pancreatic tissue homeostasis. 
Macrophages turned out to be another cell type expressing Wnt/ $\beta$-catenin pathway-associated molecules in the fibrotic stroma of human pancreatic specimens. Macrophages are known to play important regulatory roles in ontogeny and adult tissue homeostasis [41]. They display great diversity in phenotype and function in response to variable microenvironmental signals and are intimately involved in tissue repair and the development and progression of pathologic conditions such as cancer, inflammatory diseases and fibrosis [41]. In fibroses, not only pro-fibrotic but also anti-fibrotic activities for regulatory macrophages have been identified. In chronic pancreatitis macrophages have been suggested to promote fibrosis via cross-talk with PSCs [42]. They have also emerged as active players in the progression of acute pancreatitis [43].

Among other regulatory molecules, Wnts are known to be delivered by macrophages upon tissue injury to facilitate homeostatic repair and regeneration [41]. In the process Wnts enhance myofibroblast activation and deposition of extracellular matrix components. In macrophages themselves $\mathrm{Wnt} / \beta$-catenin signalling has been shown to regulate polarization [44] and to promote motility and adhesion [45]. According to our present data, macrophages appear to be major contributors of Wnt ligands and SFRP4 to the microenvironment of pancreatic fibrosis. The expression and diverse regulatory functions of molecules related to $\mathrm{Wnt} / \beta$-catenin signalling in macrophages have previously been described in a variety of other organs, including the intestine [46], kidney [44] and liver [47].

In conclusion, our study showed distinct cellular and subcellular alterations in the expression of $\beta$-catenin, Wnt2, Wnt5a and SFRP4 along with increased fibrosis in human pancreatic tissue. Some of the alterations could be associated with acinar-PSC cross-talk in the mouse co-culture setting, rendering the culture system a relevant tool for studying pancreatic Wnt/ $\beta$-catenin signalling in vitro. Our novel observation of an abundance of immunoreactive SFRP4 in acinar cells in addition to PSCs warrants further studies on its regulatory activities in pancreatic tissue. The introduction of macrophages as another source of $\mathrm{Wnt} / \beta$-catenin pathway-associated proteins in fibrotic pancreata emphasizes the central role of the signalling system in pancreatic pathophysiology.

\section{Acknowledgements}

We thank Niina Ikonen for expert technical assistance. This study was financially supported by the Sigrid Jusélius Foundation, Finland and the Medical Research Fund of Pirkanmaa Hospital district, Finland. The authors have nothing to disclose. 


\section{References}

1. Nelson WJ, Nusse R. Convergence of Wnt, $\beta$-catenin, and cadherin pathways. Science 2004; 303: 1483-7.

2. Saito-Diaz K, Chen TW, Wang X, Thorne CA, Wallace HA, Page-McCaw A, et al. The way Wnt works: Components and mechanism. Growth Factors 2013; 31: 1-31.

3. Adams PD, Enders GH. Wnt signaling and senescence: a tug of war in early neoplasia? Cancer Biol Ther 2008; 7: 1706-11.

4. Lam AP, Gottardi CJ. $\beta$-catenin signaling: a novel mediator of fibrosis and potential therapeutic target Curr Opin Rheumatol 2011; 23: 562-7.

5. Kam Y, Quaranta V. Cadherin-bound beta-catenin feeds into the Wnt pathway upon adherens junctions dissociation: evidence for an intersection between beta-catenin pools. PLoS One 2009; 4:e4580. doi: 10.1371/journal.pone.0004580.

6. Mikels AJ, Nusse R. Purified WNT5a protein activates or inhibits beta catenin-TCF signalling depending on receptor context. PLoS Biol 2006; 4: e115.

7. Grumolato L, Liu G, Mong P, Mudbhary R, Biswas R Arroyave R, et al. Canonical and noncanonical Wnts use common mechanisms to activate completely unrelated coreceptors. Genes Dev 2010; 24: 2517-30.

8. Sato A, Yamamoto H, Sakane H, Koyama H, Kikuchi A. Wnt5a regulates distinct signalling pathways by binding to Frizzled 2. EMBO J 2010; 29:41-54.

9. Cruciat CM, Niehrs C. Secreted and transmembrane wnt inhibitors and activators. Cold Spring Harb Perspect Biol 2013; 5:a015081. doi: 10.1101/cshperspect.a015081.

10. Bovolenta P, Esteve P, Ruiz JM, Cisneros E, Lopez-Rios J. Beyond Wnt inhibition: new functions of secreted frizzled-related proteins in development and disease. J Cell Sci 2008; 121: 737-46.

11. Heller RS, Klein T, Ling Z, Heimberg H, Katoh M, Madsen OD, et al. Expression of Wnt, Frizzled, sFRP, and DKK genes in adult human pancreas. Gene Expr 2003; 11:141-7.

12. Keefe MD, Wang H, De La O J-P, Khan A, Firpo MA, Murtaugh C. $\beta$-catenin is selectively required for the expansion and regeneration of mature pancreatic acinar cells in mice. Disease Models \& Mechanisms 2012; 5: 503-14.

13. Jiang H, Li Q, He C, Li F, Sheng H, Shen X, et al. Activation of the Wnt pathway through Wnt2 promotes metastasis in pancreatic cancer. Am J Cancer Res 2014; 4: 537-44. 
14. Arensman MD, Kovochich AN, Kulikauskas RM, Lay AR, Yang PT, Li X, et al. WNT7B mediates autocrine Wnt/ß-catenin signaling and anchorage-independent growth in pancreatic adenocarcinoma. Oncogene 2014; $33: 899-908$.

15. Arensman MD, Nguyen P, Kershaw KM, Lay AR, Oatertag-Hill CA, Sherman MH, et al. Calcipotriol Targets LRP6 to Inhibit Wnt Signaling in Pancreatic Cancer. Mol Cancer Res 2015; 13:1509-19.

16. Froeling FE, Feig C, Chelala C, Dobson R, Mein CE, Tuveson DA, et al. Retinoic acid-induced pancreatic stellate cell quiescence reduces paracrine Wnt- $\beta$-catenin signaling to slow tumor progression. Gastroenterology 2011; 141:1486-97.

17. Sano M, Driscoll DR, DeJesus-Monge WE, Quattrochi B, Appleman VA, Ou J, et al. Activation of WNT/ $\beta$ catenin signaling enhances pancreatic cancer development and the malignant potential via up-regulation of Cyr61. Neoplasia 2016; 18:785-94.

18. Carbone C, Piro G, Gaianigo N, Ligorio F, Santoro R, Merz V, et al. Adipocytes sustain pancreatic cancer progression through a non-canonical WNT paracrine network including ROR2 nuclear shuttling. Int J Obes (Lond) 2018; 42:334-43.

19. Carapuca EF, Gemenezidis E, Feig C, Bapiro TE, Williams MD, Wilson AS, et al. Anti-stromal treatment together with chemotherapy targets multiple signaling pathways in pancreatic adenocarcinoma. J Pathol 2016; 239: 286-96.

20. Apte MV, Pirola RC, Wilson JS. Pancreatic stellate cells: a starring role in normal and diseased pancreas. Front Physiol 2012; 3:344.

21. Hu Y, Wan R, Yu G, Shen J, Ni J, Yin G, et al. Imbalance of Wnt/Dkk Negative feedback promotes peristent activation of pancreatic stellate cells in chronic pancreatitis. PLoS One 2014; 9: e95145.

22. Xiao W, Jiang W, Shen J, Yin G, Fan Y, Wu D, et al. Retinoic acid ameliorates pancreatic fibrosis and inhibits the activation of pancreatic stellate cells in mice with experimental chronic pancreatitis via suppressing the Wnt/ $\beta$-catenin signaling pathway. PLoS One 2015; 10:e141462.

23. Ma B, Hottiger MO. Crosstalk between Wnt/ $\beta$-Catenin and NF- $\mathrm{B}$ S Signaling Pathway during Inflammation. Front Immunol 2016; 7:378.

24. Jian H, Shen X, Liu I, Semenov M, He X, Wang X-F. Smad3-dependent nuclear translocation of $\beta$-catenin is required for TGF- $\beta 1$-induced proliferation of bone marrow-derived adult human mesenchymal stem cells. Genes Dev. 2006; 20: 666-674. 
25. Gukovsky I, Gukovskaya A. Nuclear factor-кB in pancreatitis: Jack-of-all-trades, but which one is more important? Gastroenterology 2013; 144: 26-9.

26. Ahmed S, Bradshaw A-D, Gera S, Dewan MZ, Xu R. The TGF- $\beta$ /Smad4 Signaling Pathway in Pancreatic Carcinogenesis and Its Clinical Significance. J Clin Med 2017; 6:5. doi: 10.3390/jcm6010005.

27. Pang TCY, Wilson JS, Apte MV. Pancreatic stellate cells: what's new? Curr Opin Gastroenterol 2017; 33: 36673.

28. Bläuer M, Laaninen M, Sand J, Laukkarinen J. Reciprocal stimulation of pancreatic acinar and stellate cells in a novel long-term in vitro co-culture model. Pancreatology 2016; 16: 570-7.

29. Bläuer M, Nordback I, Sand J, Laukkarinen J. A novel explant outgrowth culture model for mouse pancreatic acinar cells with long-term maintenance of secretory phenotype. Eur J Cell Biol 2011; 90: 1052-60.

30. Bläuer M, Sand J, Laukkarinen J. Cryopreserved mouse pancreatic acinar cells from long-term outgrowth cultures maintain their secretory phenotype after thawing. Pancreatology 2013; 13: 610-4.

31. Murtaugh LC, Keefe M. Regeneration and Repair of the Exocrine Pancreas. Annu Rev Physiol 2015; 77: 22949.

32. Dietrich C, Scherwat J, Faust D, Oesch F. Subcellular localization of $\beta$-catenin is regulated by cell density. BBRC 2002; 292: 195-9.

33. Howard S, Deroo T, Fujita Y, Itasaki N. A positive role of cadherin in Wnt/ $\beta$-catenin signalling during epithelial-mesenchymal transition. PLoS ONE 2011; 6: e23899.

34. Bachmann IM, Straume O, Puntervoll HE, Kalvenes MB, Akslen LA. Importance of P-Cadherin, $\beta$-Catenin, and Wnt5a/Frizzled for Progression of Melanocytic Tumors and Prognosis in Cutaneous Melanoma. Clin Cancer Res 2005; 11: 8606-14.

35. Pizzatti L, Binato R, Cofre H, Gomes BE, Dobbin J, Haussmann ME, et al. SUZ12 is a candidate target of the non-canonical WNT pathway in the progression of chronic myeloid leukemia. Genes, Chromosomes \& Cancer 2010; 49: 107-18.

36. Prgomet Z, Andersson T, Linberg P. Higher expression of WNT5A protein in oral squamous cell carcinoma compared with dysplasia and oral mucosa with a normal appearance. Eur J Oral Sci 2017; 125: 237-46.

37. Kordes C, Sawitza I, Häussinger D. Hepatic and pancreatic stellate cells in focus. Biol Chem 2009; 390: 100312.

38. Monga SP. $\beta$-catenin signaling and roles in liver homeostasis, injury, and tumorigenesis. Gastroenterology 2015; 148: 1294-310. 
39. Bu XM, Zhao CH, Zhang N, Gao F, Lin S ,Dai XW. Hypermethylation and aberrant expression of secreted frizzled-related protein genes in pancreatic cancer. World J Gastroenterol 2008; 14: 3421-4.

40. Pohl S, Scott R, Arfuso F, Perumal V, Dharmarajan A. Secreted frizzled-related protein 4 and its implications in cancer and apoptosis. Tumor Biol 2015; 36: 143-52.

41. Wynn TA, Vannella KM. Macrophages in tissue repair, regeneration, and fibrosis. Immunity 2016; 44:45062.

42. Xue J, Sharma V, Hsieh MH, Chawla A, Murali R, Pandol SJ, et al. Alternatively activated macrophages promote pancreatic fibrosis in chronic pancreatitis. Nat Commun 2015; 6: 7158.

43. Shrivastava P, Bhatia M. Esssential role of monocytes and macrophages in the progression of acute pancreatitis. World J Gastroenterol 2010; 16:3995-4002.

44. Feng Y, Ren J, Gui Y, Wei W, Shu BB, Lu Q, et al. Wnt/ $\beta$-catenin-promoted macrophage alternative activation contributes to kidney fibrosis. J Am Soc Nephrol 2018; 29: 182-93.

45. Amini-Nik S, Cambridge E, Yu W, Guo A, Whetstone H, Nadesan P, et al. $\beta$-catenin-regulated myeloid cell

46. Saha S, Aranda E, Hayakawa Y, Bhanja P, Atay S, Brodin NP, et al. Macrophage-derived extracellular vesiclepackaged WNTs rescue intestinal stem cells and enhance survival after radiation injury. Nat Commun 2016; 7:13096. doi: 10.1038/ncomms13096.

47. Boulter L, Govaere O, Bird TG, Radulescu S, Ramachandran P, Pellicoro A, et al. Macrophage-derived Wnt opposes Notch signaling to specify hepatic progenitor cell fate in chronic liver disease. Nat Med 2012; 18: $572-9$. 


\section{Figure legends}

Figure 1. Wnt/ $\beta$-catenin pathway-associated proteins in human pancreatic tissue. The picture shows immunoreactivity of $\beta$-catenin, Wnt2, Wnt5a and SFRP4 in representative specimens of non-fibrotic $(a-d)$, moderately fibrotic $(e-h)$ and highly fibrotic $(i-l)$ tissue areas. Arrows in $(a-h)$ indicate immunoreactivity in representative acinar cells. In ( $i-l)$ arrows and arrowheads point at immunopositive fibroblastoid/stellate cells and macrophages, respectively. Open arrow in $(i)$ indicates positive expression in acinar cells. Bars $50 \mu \mathrm{m}$. IL= islet of Langerhans.

Figure 2. Wnt/ $\beta$-catenin pathway-associated proteins in mono- and co-cultures of mouse pancreatic acinar cells and PSCs. Acinar cells $(a-h)$ and PSCs $(i-p)$ were maintained in parallel mono- and co-cultures for 4 days and thereafter subjected to immunocytochemical analyses of $\beta$-catenin, Wnt2, Wnt5a and SFRP4. Representative immunopositive cells are indicated with arrows. Bars $50 \mu \mathrm{m}$; in $a$ and $d 25 \mu \mathrm{m}$.

Figure 3. Schematic representation of the expression of $\beta$-catenin, Wnt2, Wnt5a and SFRP4 in human pancreatic tissue samples $(a-c)$ and in mono-and co-cultures of mouse acinar cells and PSCs in vitro (d-f). The expression pattern of the studied proteins was closely similar in acini in the non-fibrotic tissue microenvironment $(a)$ and in acinar cell monocultures $(d)$, with an intense membrane expression of $\beta$-catenin. The interacinar stroma in non-fibrotic tissue was immunonegative (a). Prominent subcellular changes compared to $(a)$ and $(d)$ were detected in the acinar cell expression of the proteins in moderately fibrotic tissue areas $(b)$ and in acinar/PSC co-cultures $(e)$. Note the nuclear location of $\beta$ catenin and $\mathrm{Wnt5a}$ in $(b)$ and the nuclear expression of Wnt5a and perinuclear distribution $\beta$-catenin and SFRP4 in (e). Stromal cells in moderately fibrotic tissue remained immunonegative (b). All proteins were detected in the cytoplasmic compartment of co-cultured PSCs $(e)$. In acinar cells surrounded by dense fibrosis immunoreactivities were weak or absent $(c)$ whereas stromal fibrobastoid/stellate cells and macrophages expressed varying intensities of all the proteins studied. Nuclear expression of $\beta$-catenin was displayed in a subset of stromal fibrobastoid/stellate cells (c) as well as in a number of monocultured PSCs (f). 


\section{FIGURE 1}

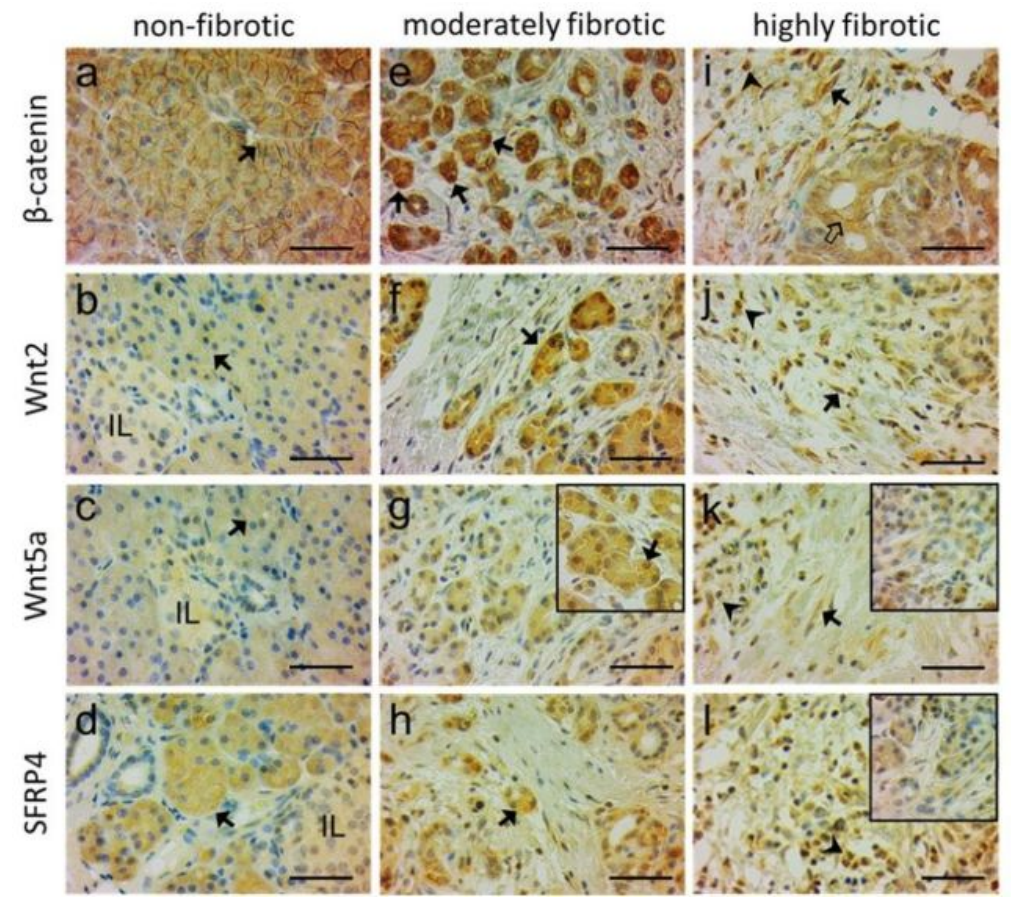




\section{FIGURE 2}

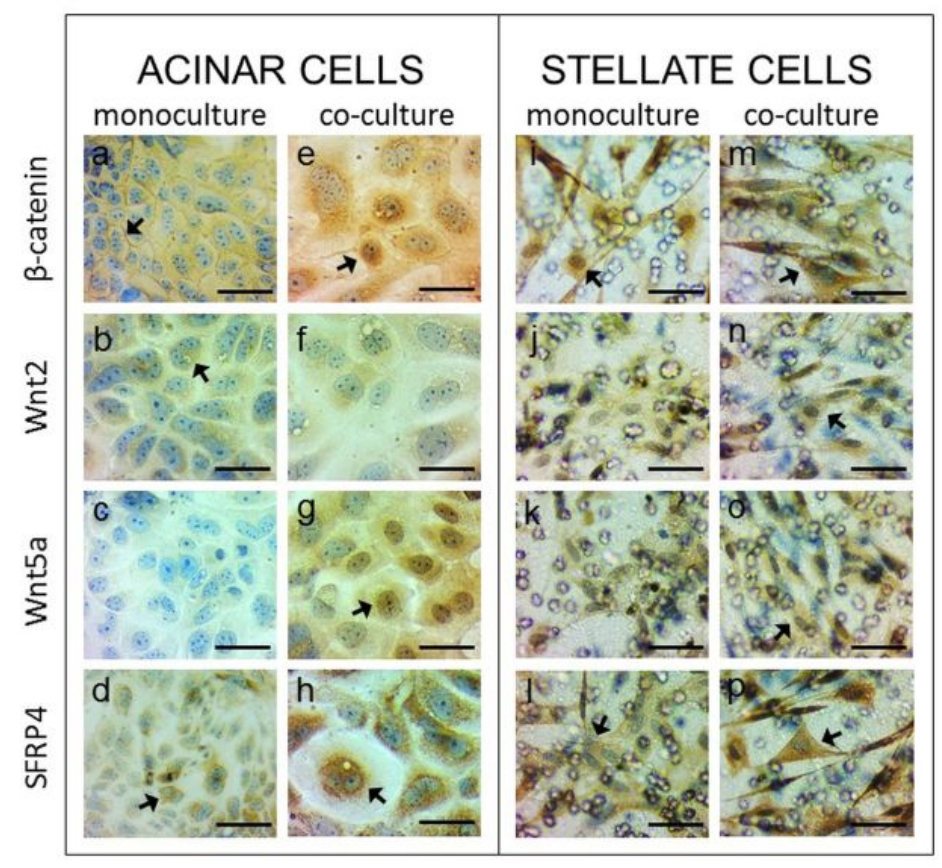




\section{FIGURE 3}

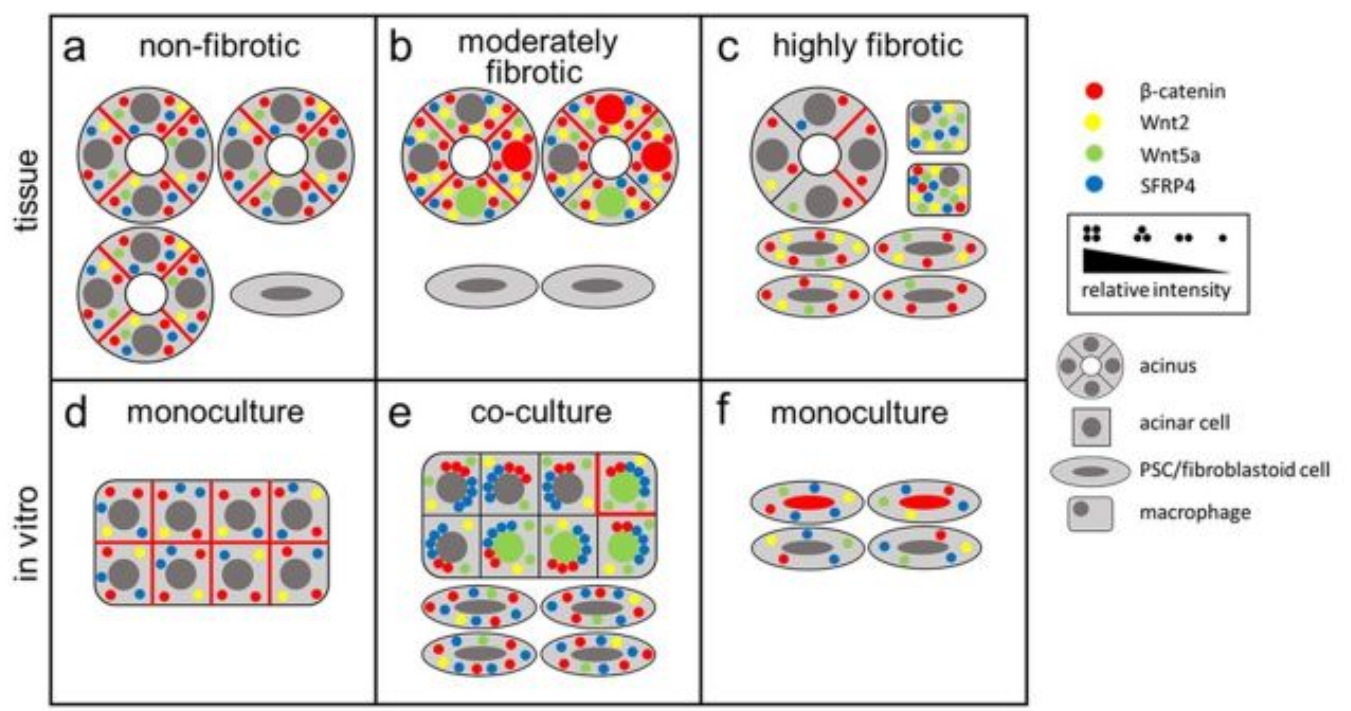




\section{SUPPLEMENT 1}

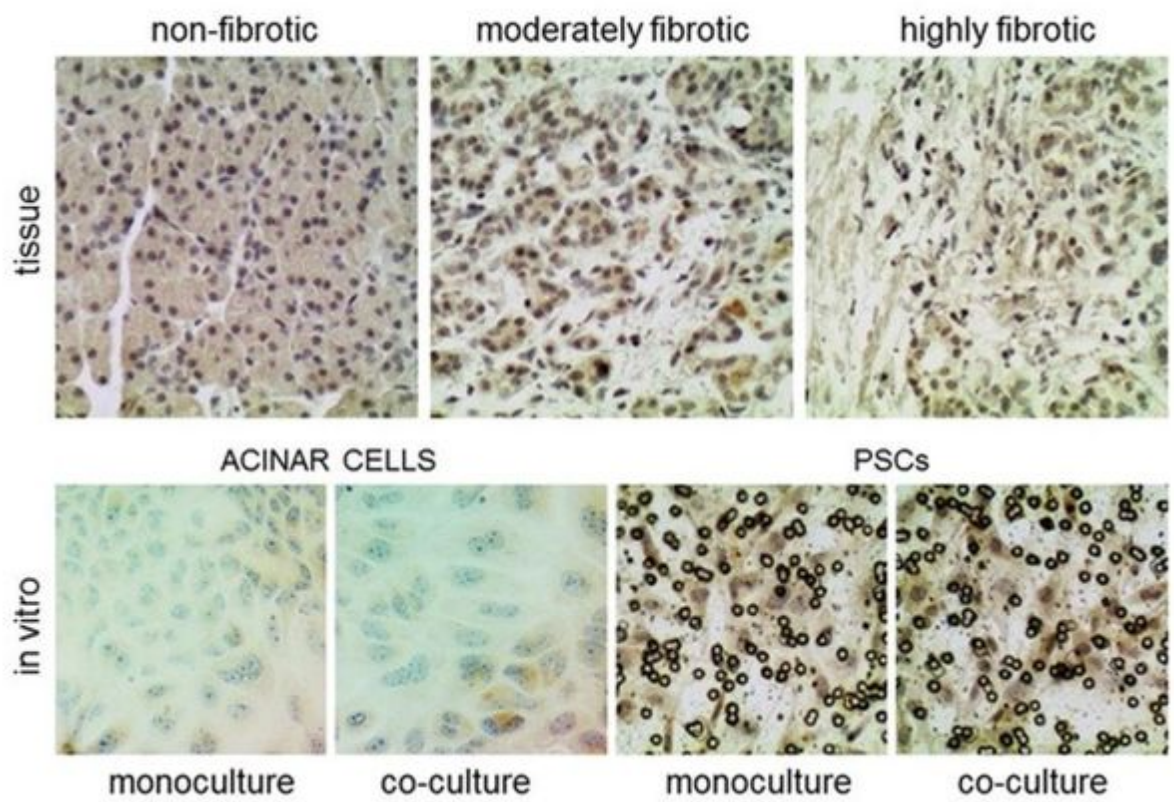

S1. Negative staining controls for immunohisto- and cytochemistry. Human tissue samples and mouse acinar cells and PSCs were subjected to the staining protocol with antibody dilution buffer in place of primary antibodies. The staining was performed with the Histostain Plus Broad Spectrum Kit (Life Technologies, Frederick, MD) followed by DAB-based chromogenic detection (DAB Substrate Kit, Thermo Fisher Scientific, Rockford, II). 
SUPPLEMENT 2
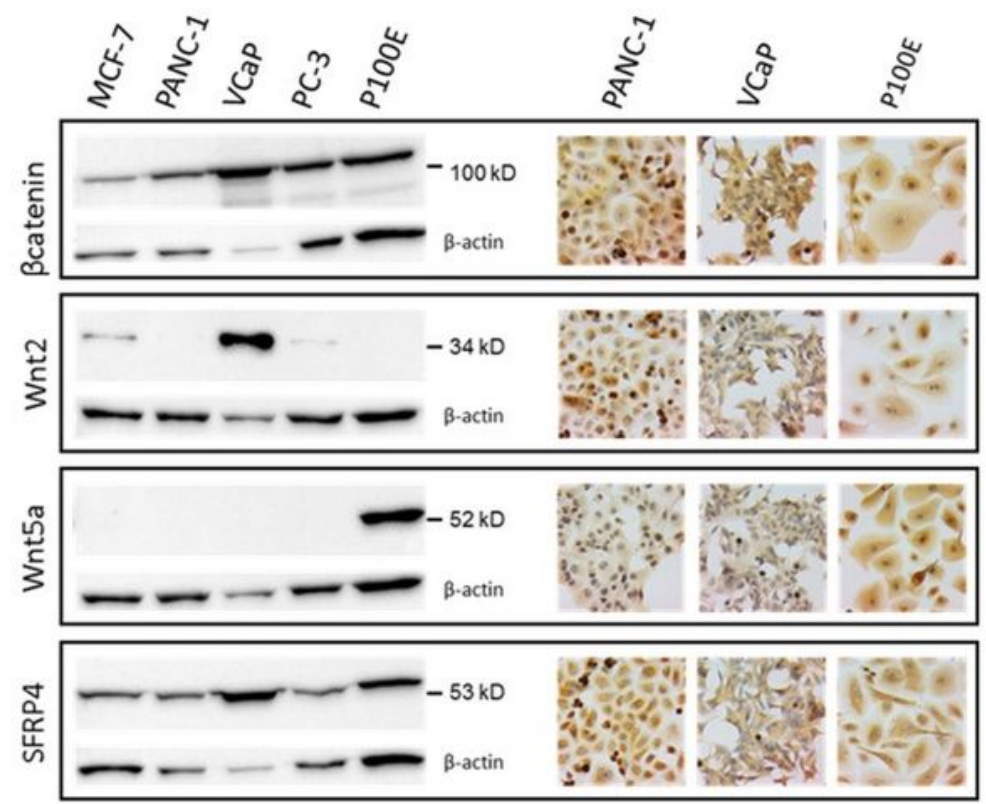

control stainings with secondary antibody only

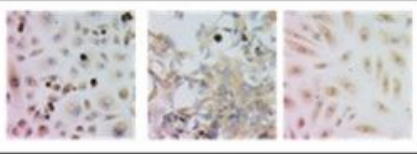

S2. Antibody validation. Four human cancer cell lines (MCF-7, PANC-1, PC-3, VCaP) and the primary cell line P100E derived from human prostatic hyperplasia were used to evaluate the antibodies against $\beta$-catenin (ab16051), Wnt2 (ab109222), Wnt5a (PA5-72454) and SFRP4 (ab154167). The antibodies were shown to detect their corresponding antigens in Westem blots performed with $20 \mu \mathrm{g}$ protein/well and in cells subjected to immunocytochemical staining. Antibody dilutions in Westem blot analysis were 1:2000 ( $\beta$-catenin), 1:1000 (Wnt2, SFRP4) and 1:500 (Wnt5a) and in immunocytochemical analysis 1:100 ( $\beta$-catenin, Wnt2, SFRP4) and 1:500 (WNT5a). 


\section{SUPPLEMENT 3}
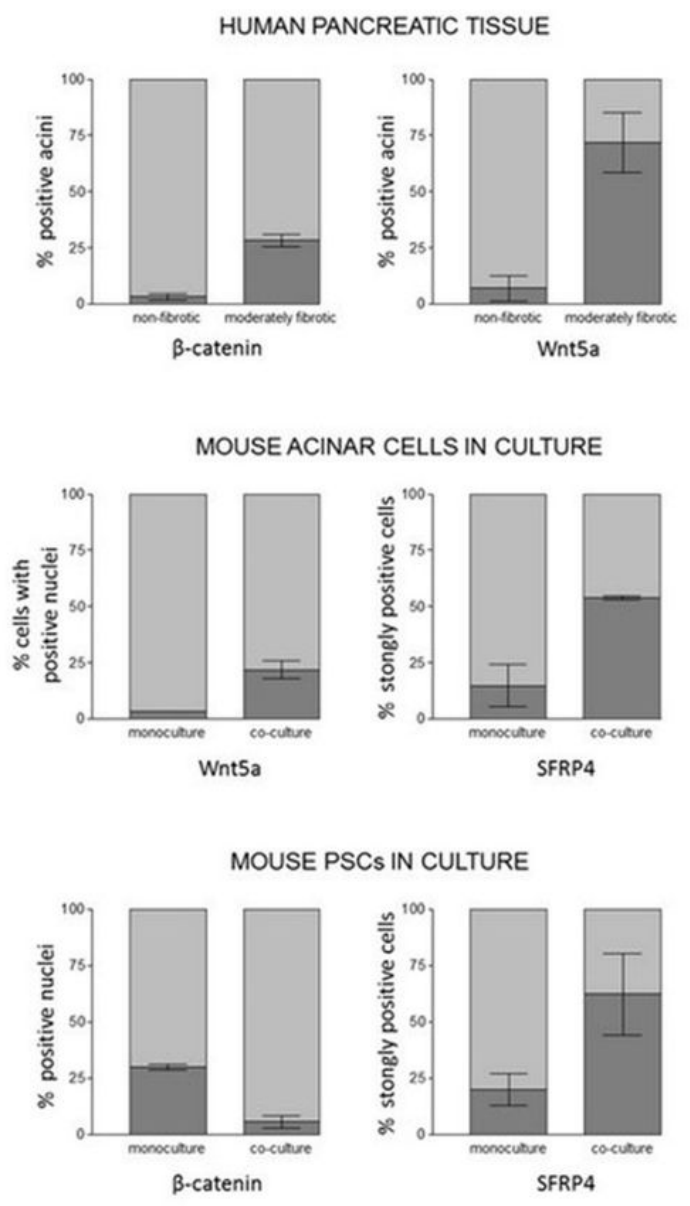

S3. Quantitation of immunohisto- and cytochemical stainings. The bars represent the mean percentage $=$ SEM of six randomly picked microscopic areas of tissue or cells in culture (200x magnification). In human tissues, acini showing at least one immunopositive cell were regarded as positive. 